\title{
Cáncer de cervix IB. Análisis de 110 casos
}

\author{
HOSPITAL UNIVERSITARIO SAN VICENTE DE PAUL - MEDELLIN
}

Dres.: Elmer Pinilla Galvis*, Jaime Uribe Duque* y Germán González**

\section{INTRODUCGION}

En el trabajo Cáncer de Cervix Uterino IB: Análisis de 290 casos (1), que presentamos durante el XVI Congreso Colombiano de Ginecología y Obstetricia efectuado en Cali en diciembre de 1985, hicimos notar un hecho importante en relación con el comportamiento del cáncer cervical en la Clínica de Tumores de HUSVP de Medellín: una notable mejoría en la proporción de los estadios precoces $(0+\mathrm{I})$ frente a la de los estadios avanzados (II + III + IV). En los años 50 s, la proporción era crítica: los precoces eran el 13\%, y los avanzados el $87 \%$ (2). La Gráfica 2. muestra que, de un total de 3.200 casos de cáncer de cervix entre 01-01-70 y 31-12-84, los precoces representaban el $45 \%$ y los avanzados el $55 \%$; y entre 1.025 habidos en el período 01-01-85 y $30-06-87$, los precoces son el $49.8 \%$ y los avanzados el $50.2 \%$.

\section{MATERIAL Y METODO}

Los datos obtenidos para el presente trabajo fueron extraídos del registro computarizado de las historias clínicas de las pacientes nuevas que acudieron a la Clínica de Tumores entre 01-07-85 y 30 06-87, período durante el cual se diagnosticaron 110 casos nuevos de cáncer de cervix uterino IB según la clasificación de la FIGO. Las pacientes pro-

* Profesor Titular, Facultad de Medicina Universidad de Antioquia - Medellín.

** Profesor Titular, Escuela de Salud Pública - Medellín.
GRAFICO 2

PERIODOS

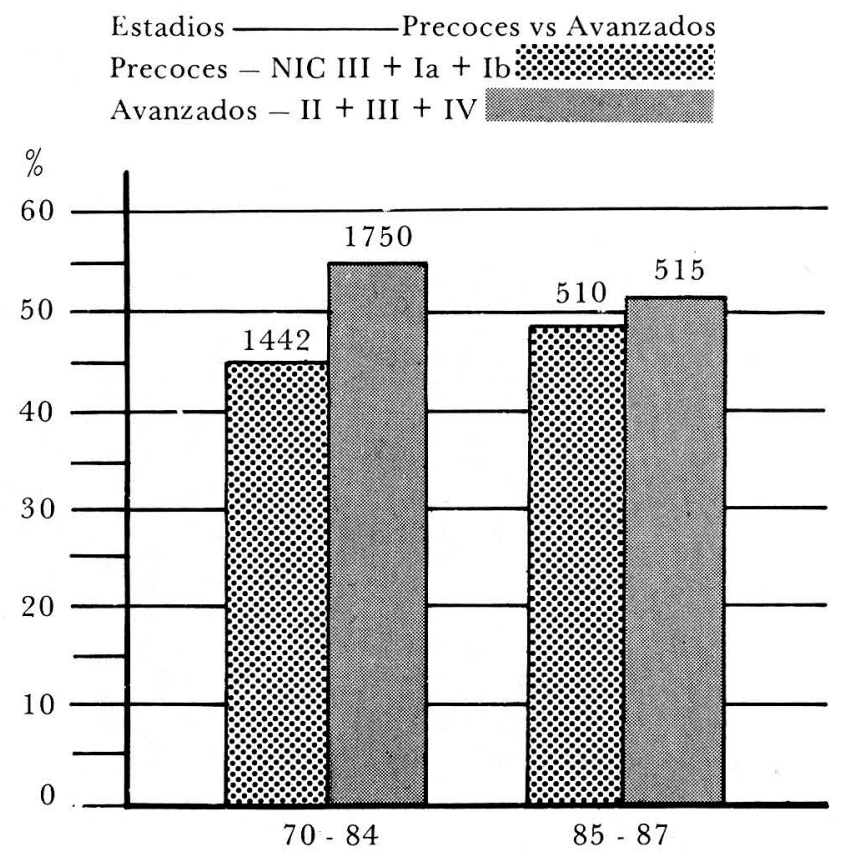

cedían del área metropolitana de Medellín y del resto del Departamento de Antioquia principalmente, pero también de los Departamentos de Caldas, Chocó, Sucre y Córdoba. El manejo de los casos estuvo a cargo de un grupo multidisciplinario compuesto por ginecólogos, radioterapeutas y patólogos que se ha mantenido casi invariable desde $1970 \mathrm{y}$ con una positiva unidad de criterios diagnósticos y terapéuticos. 


\section{Descripción}

A pesar de que el estadio IB se ha mantenido en el cuarto lugar entre los demás estadios desde 1970, después del IIIB, del 0 y del IIB, su representación porcentual ha aumentado así: $7.2 \%$ en el período 70-82 (parte infero-izquierda del cuadro); $9.2 \%$ en el $83-84$, y $10.5 \%$ en el $85-87$. Mientras tanto, el estadio 0 ha disminuido casi $10 \%$ y los demás se han mantenido más o menos estables en los dos últimos períodos. Adicionalmente se nuede observar, como lo señalamos en el trabajo presentado en Cali, que los subgrupos A de cada estadio tienen una representación muy baja en relación con los subgrupos B respectivos.

\section{CUADRO 1}

\section{DISTRIBUCION PORCENTUAL POR ESTADIOS SEGUN PERIODOS}

\begin{tabular}{|c|r|r|r|r|}
\hline Estadio & No. & \multicolumn{1}{|c|}{$\%$} & No. & \multicolumn{1}{c|}{$\%$} \\
\hline 0 & 387 & 44.4 & 427 & 94.7 \\
\hline IA* & 25 & 2.8 & 52 & 4.2 \\
\hline IIB & 81 & 9.2 & 129 & 10.5 \\
IIA* & 4 & 0.4 & 16 & 1.3 \\
IIB & 113 & 12.9 & 172 & 14.4 \\
IIIA* & 2 & 0.2 & 3 & 0.2 \\
IIIB & 251 & 28.7 & 409 & 31.9 \\
IV & 11 & 1.2 & 17 & 1.4 \\
\hline Total & 874 & 100.0 & 1225 & 100.0 \\
\hline
\end{tabular}

$$
70-83 \text { Junio }=310
$$

Aumento numérico

Además de su aumento porcentual, el estadio IB muestra un sorprendente aumento numérico. Según los períodos contemplados en la Gráfica, dicho aumento fue así: 96 en el 70-74; 108 en el 75$79 ; 168$ en el 80-84, y 129 en el 85-87. Esto muestra que en los dos últimos años se ha diagnosticado más del $75 \%$ de lo que se hizo en los 4 años anteriores (168). (Gráfico 3).

Si se hace un cálculo de proyección a 1989, a partir de 1985, tendremos en aquel año 210 nuevos casos de cáncer de cervix IB. Sin embargo, tal parece que el ritmo de aumento ha principiado a
GRAFICO 3

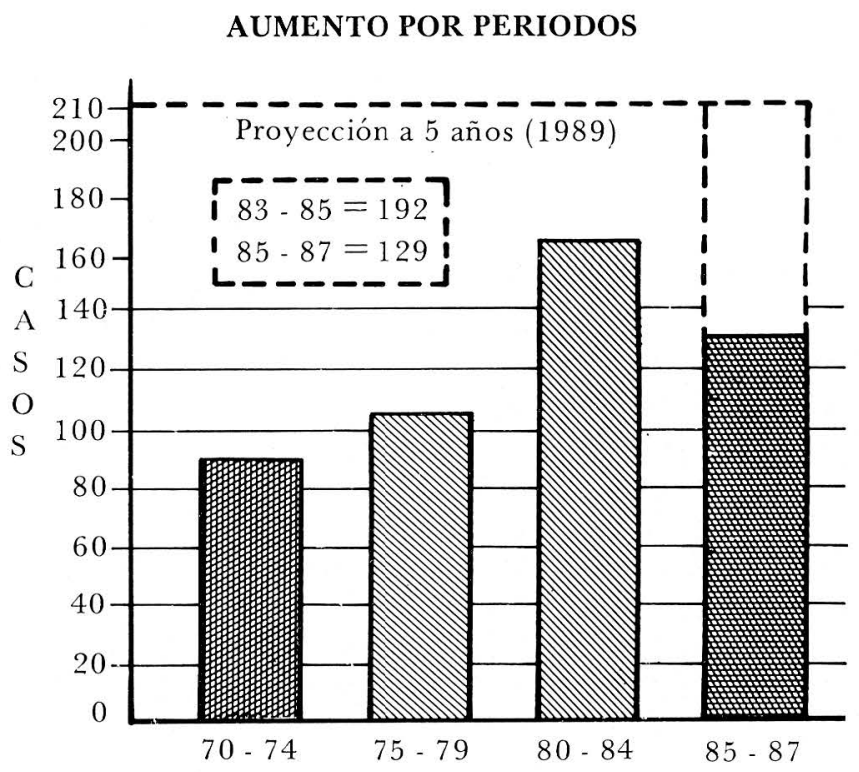

aminorar, como se observa en el recuadro de la Gráfica, al comparar los 192 casos entre 01-07-83 y 30-06-85 y los 129 entre 01-07-85 y 30-06-87.

\section{GRAFICO 4}

\section{DISTRIBUCION POR EDADES}
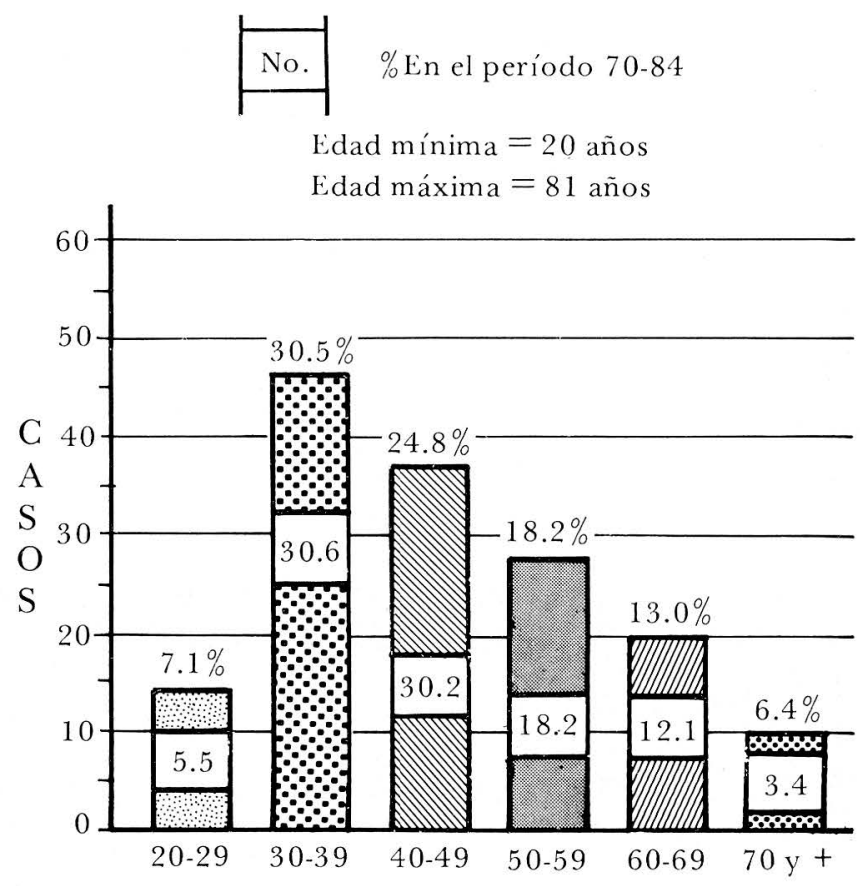


\section{Distribución por edades}

En la Gráfica 4 se advierte la ausencia del estadio IB en mujeres menores de 20 años entre los 110 casos. Tampoco los hubo entre el total de 501 entre 1970 y 1984. La mayor concentración de casos se encuentra entre los 30 y los 49 años que hacen entre los dos grupos el $54 \%$. Dentro de cada barra se encuentran los porcentajes respectivos del período 70-84 del trabajo presentado en Cali para su comparación con el actual, que nos muestran una disminución de $5.4 \%$ en el grupo 40-49.

\section{Embarazos}

Entre los 110 casos hubo 3 embarazos, es decir, $2.7 \%$, cifras similares a la del período $70-84$ (2.1\%) y a las de la literatura nacional $(1,3,4$ y 5$)$. Dos embarazos del primer trimestre fueron tratados con histerectomía radical con feto in situ, y uno con anexohisterectomía solamente con feto in situ.

\section{Tratamiento}

En el Cuadro 2 se advierte que, de los $110 \mathrm{ca-}$ sos, a 36 se les trató con histerectomía radical; a 33 con radioterapia $\left(\mathrm{Co}+\mathrm{C}^{\mathrm{S}}\right)$; a 23 , histerectomía simple más Co; a 5 , sólo histerectomía simple; 4 fueron tratadas con histerectomía radical más Co; 2 , con histerectomía vaginal más Co; 2 , con histerectomía vaginal solamente; 3 , con solamente conización; 1 recibió laparotomía exploradora, y 1 estaba sin tratamiento en el momento del corte.

\section{CUADRO 2}

TRAT AMIENTO

\begin{tabular}{|c|c|c|}
\hline Método & Número & $\%$ \\
\hline Radioterapia sola (Co $+\mathrm{Ce})$ & 33 & 30.0 \\
\hline $\begin{array}{l}\text { Hist. Rad. + Limfadenectomia pélvica } \\
\text { Histerectomia simple + Cobalto }\end{array}$ & $\begin{array}{l}36 \\
23\end{array}$ & $\begin{array}{l}32.7 \\
20.8\end{array}$ \\
\hline $\begin{array}{l}\text { Histerectomía simple sola } \\
\text { Hist. Rad. + Linfadenectomía pélvica + Cobalto } \\
\text { Hist. vaginal + Cobalto } \\
\text { Hist. vaginal sola } \\
\text { Otro tratamiento }\end{array}$ & $\begin{array}{l}5 \\
4 \\
2 \\
2 \\
5\end{array}$ & 4.6 \\
\hline Total & 110 & \\
\hline
\end{tabular}

\section{Compromiso ganglionar}

De las 36 histerectomías radicales, 4 mostraron compromiso de los ganglios pélvicos, lo que da un $11.1 \%$, cifras que son similares a las del trabajo anterior y a las de la literatura nacional (3, 4 y 5$)$.

\section{Estado actual}

Desde 01-01-70 hasta 30-06-87, han habido en la Clínica de Tumores del HUSVP de Medellín 501 casos de cáncer de cervix IB. En la Gráfica 5 se ve, de manera global, que están vivas 269 (53.7), perdidas $185(36.9 \%)$ y muertas $47(9.4 \%)$. Con la mejoría de los sistemas de registro y de seguimiento y pesquisa de las pacientes, se advierte también una mejor distribución de los tres acápites.

\section{GRAFICO 5}

\section{ESTADO ACTUAL}

\section{CASOS}

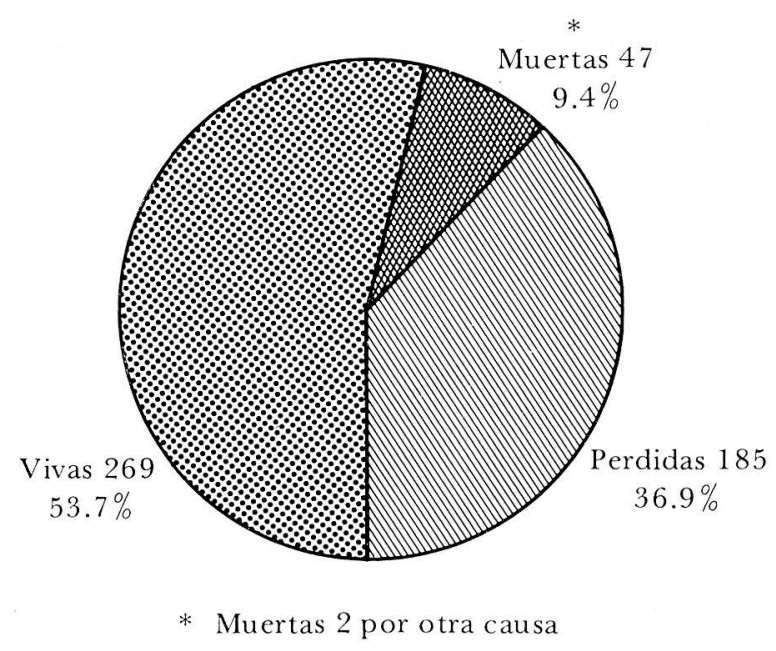

Probabilidad de sobrevida

La Gráfica 6 muestra la probabilidad de sobrevida de los estadios IB en el período 70-79; las mujeres menores de 40 años tenían $85 \%$ de probabilidades de estar vivas a los 10 años de seguimiento, contra $80 \%$ de los mayores de esa edad y muestra lo mismo en el período $80-87$ para 8 años de seguimiento: $92 \%$ en menores de 40 años y $85 \%$ en mayores de esa edad. 
GRAFICOS 6 y 7

\section{PROBABILIDAD DE SOBREVIDA}

MEDELLIN COLOMBIA

$1970-1979$

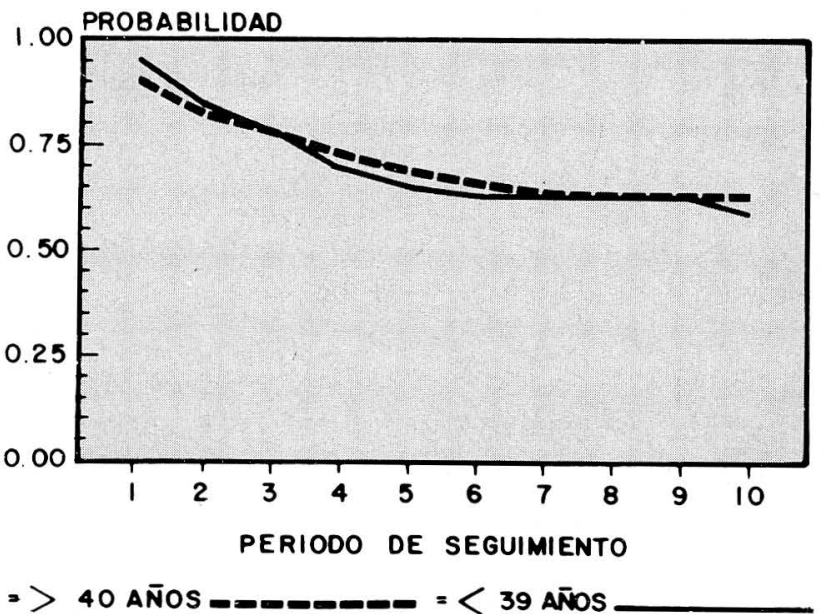

\section{DISCUSION}

De los informes del presente trabajo, resaltan varios hechos:

1. Una satisfactoria y esperanzadora redistribución numérica y porcentual en favor de los estadios Precoces del cáncer del cervix uterino frente a los avanzados. La citología vaginal sistemáticamente periódica y el advenimiento de la colposcopia y de la biopsia dirigida por ella, han mejorado sustancialmente el diagnóstico de los estadios NIC, IA y IB que, una vez tratados convenientemente, dejan de engrosar los estadíos avanzados, por lo cual tendrán que disminuir. El hecho de que en la Clínica de Tumores del HUSVP, que atiende una amplia zona del país, los estadios precoces están a punto de superar los avanzados, nos lleva a suponer que lo mismo esté sucediendo en el resto de Colombia, y nos hace deducir que, en un futuro no lejano, el cáncer del cervix avanzado dejará de ser una de las primeras causas de muerte femenina por cáncer, lo cual da un mentís a quienes opinan que nuestra Patria carece en absoluto de signos de progreso.

2. Un sorprendente aumento porcentual y numérico del estadío IB. Volvemos a insistir en el valor de la citología vaginal sistemáticamente periódica, en la colposcopia y en la biopsia dirigida por ella $(7,8)$. Estos dos últimos métodos diagnósticos se realizan en Medellín no sólo en la Clínica de Tumores del HUSVP, sino en el Hospital General. En Caprecón, en Profamilia y en la Unidad Médica CES, y se está extendiendo por los consultorios particulares de los ginecólogos.

3. La ausencia de cáncer de cervix IB en 501 casos y en un lapso de 17 años y medio, entre mujeres menores de 20 años de edad. La ampliamente conocida lentitud con que progresa la enfermedad maligna del cervix hace que los estadios invasores se presenten sólo por arriba de ese límite de edad.

4. Una más baja proporción porcentual y numérica de los subgrupos $\mathrm{A}$ de cada estadio frente a los respectivos subgrupos $\mathrm{B}$, fenómeno para el cual no tenemos explicación.

5. Una baja tasa de embarazos en pacientes con cáncer de cervix IB, como ocurre en general con los demás estadios invasores, fenómeno detectado en Colombia desde 1976 (6). Aun considerando los métodos anticonceptivos, ampliamente usados en la actualidad, las mujeres con cáncer invasor del cervix son de por sí relativamente infértiles, situación que se agrava con el progreso de los estadios por la destrucción del cuello.

6. Una mejoría apreciable en los sistemas de registro de datos, y de seguimiento y pesquisa de las pacientes. 


\section{BIBLIOGRAFIA}

1. PINILlA, E. y cols. Cáncer de Cervix Uterino IB: Análisis de 290 Casos. Rev. Col. de Ginec. Obstet. Vol. 37, No. 4, 86.

2. BOTERO, J. y col. Carcinoma de Cuello Uterino en el Hospital San Vicente de Paúl de Medellín. Rev. Col. de Ginec. Obstet. 20(6): 411-415, 69 .

3. CARDONA, F. y cols. Diagnóstico y Tratamiento de las Neoplasias Intraepiteliales y de los Carcinomas Invasores Cervicales en el Embarazo. Rev. Col. de Ginec. Obstet. 32, No. 4.

4. HENAO, F. y col. 12 Años de Cirugía Radical por Cáncer de Cervix en el Hospital San Juan de Dios de Cali. Rev. Col. de Ginec. Obstet. 28(4): 150-158, 77.
5. ZUNIGA, J.E. y col. Estudio de 90 Casos de Cáncer de Cervix Uterino Tratados con Cirugía Radical. Rev. Col. de Ginec. Obstet. Vol. 36, No. 3.

6. BUENO, M. y col. Cáncer de Cervix Uterino y Cesárea a Repetición. Rev. Col. de Ginec. Obstet. Vol. 27, No. 6, 76.

7. RAMirez, F. y col. Estudio Comparativo de la Asociación de Técnicas Diagnósticas. Citología - Colposcopia. Rev. Col. de Ginec. Obstet. Vol. 37, No. 1, 86.

8. ARAGON, M.E. y col. Neoplasia Intraepitelial Cervical en el Hospital Materno Infantil., 1978-1982. Rev. Col. de Ginec. Obstet. Vol. 38, No. 2, 87.

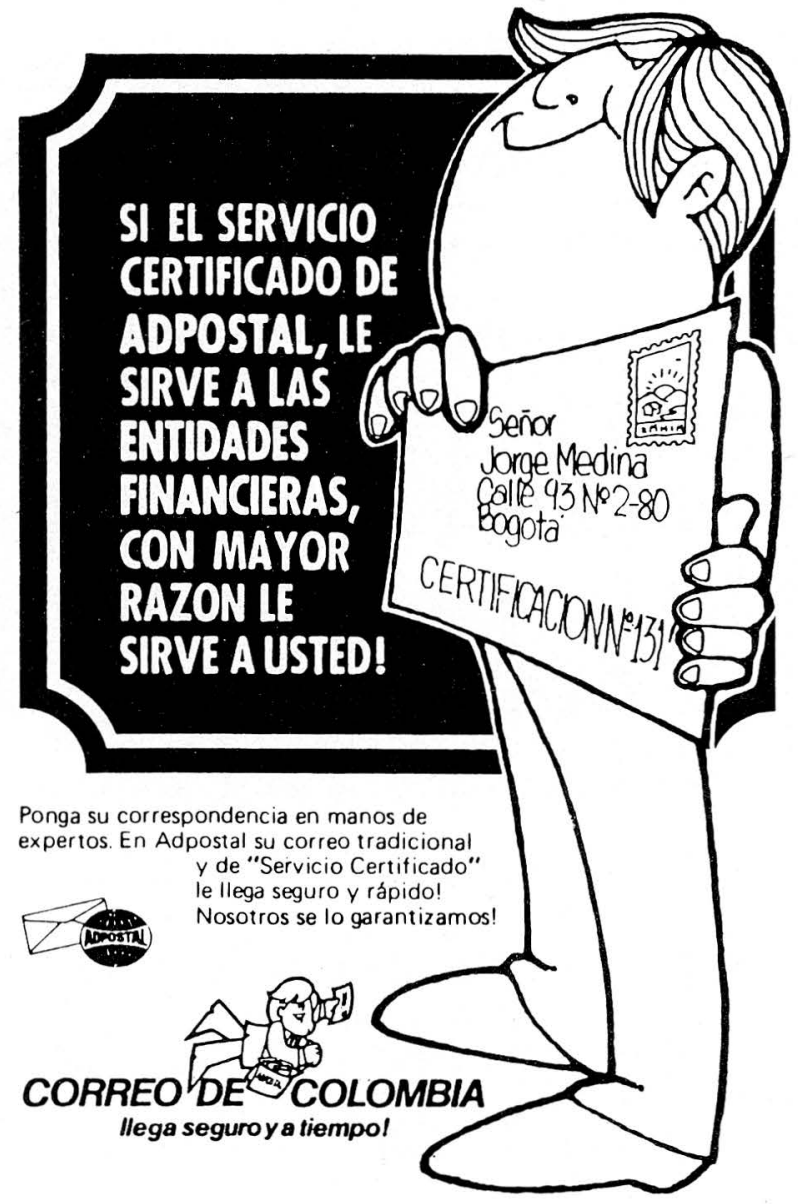

